\title{
Possibility of crystal extraction and collimation in the sub-GeV range
}

\author{
S. Bellucci ${ }^{1 * *}$ and V.M. Biryukov ${ }^{2, \dagger}$ \\ ${ }^{1}$ INFN-Laboratori Nazionali di Frascati, P.O. Box 13, 00044 Frascati, Italy \\ ${ }^{2}$ Institute for High Energy Physics, Protvino, Russia \\ (Received 25 July 2006; published 23 January 2007)
}

\begin{abstract}
Bent crystal situated in a circulating beam can serve for efficient slow extraction or active collimation of the beams. This technique, well established at $1-1000 \mathrm{GeV}$, could be efficient also at the energies as low as 0.1-1 GeV according to the computer simulations presented in this paper. Applications might include halo scraping in the spallation neutron sources or slow extraction from medical synchrotrons.
\end{abstract}

DOI: 10.1103/PhysRevSTAB.10.013501

PACS numbers: 41.85.Si, 61.85.+p

\section{INTRODUCTION}

The studies of beam extraction and collimation assisted by channeling in a bent crystal have progressed well in recent decades [1-10]. The technique was applied at up to world highest energy [4,8], in good agreement with predictions [11]. In IHEP Protvino, much of the physics program relies on crystal channeled beams used regularly since 1989 [10]. Crystal systems extract $70 \mathrm{GeV}$ protons from the IHEP main ring with efficiency of $85 \%$ at proton beam intensities of $4 \times 10^{12}$. Applications of this technique to multi-TeV range at the LHC are also considered $[12,13]$.

It would be interesting to study the feasibility of this technique also at lower energies [14], where it may assist in scraping beam halos, e.g., at the spallation neutron sources, or assist in slow extraction from multihundred $\mathrm{MeV}$ proton or light ion accelerators aimed at the production of ultrastable beams of low emittance for medical and biological applications.

Bent crystal channeling was first demonstrated at a few $\mathrm{GeV}$ in Dubna [15], and its physics first studied at 1 to $12 \mathrm{GeV}$ at Dubna, CERN, and St. Petersburg [15-17]. Crystal-based extraction from the accelerator was also first demonstrated in Dubna at 4-8 GeV [18]. More recently, efficient crystal extraction/collimation of $1 \mathrm{GeV}$ proton beam was realized in the U70 ring of IHEP Protvino [7]. So the $1-10 \mathrm{GeV}$ domain is well familiar to bent crystals.

The physics of crystal channeling is practically the same in a very broad range of energy. For heavy particles, like protons and heavy ions, this is true from the low energy in $\mathrm{KeV}$ up to the highest energy available in $\mathrm{TeV}$ range. This also holds true for a few $\mathrm{MeV}$ proton bent crystal channeling, as it has been shown in the experiments [19]. However, basic parameters of channeling vary greatly with energy. The critical bending radius is a linear function of energy. The dechanneling length grows in almost linear proportion with energy, from microns in $\mathrm{MeV}$ to meters in the $\mathrm{TeV}$ range. In a high- $\mathrm{GeV} / \mathrm{TeV}$ range, a particle beam can be

\footnotetext{
*Electronic address: bellucci@lnf.infn.it

†Electronic address: biryukov@mx.ihep.su
}

bent over a few millimeters or centimeters of crystal length. At a sub-GeV energy, the bending should be achieved over a submillimeter length.

A new invented technique of crystal bending was recently applied to produce samples with high curvature. This technique is based on the method described in Ref. [20], which was successfully applied for crystal undulator production [21]. Microscratches on a crystal surface allow us to reach high curvature of crystal bend: up to $10 \mathrm{mrad}$ over $0.5 \mathrm{~mm}$ length. In these conditions, a pure separation of channeled $500 \mathrm{MeV}$ beam with the efficiency of few tens of percent is possible.

Indeed, a first experimental demonstration of bent crystal channeling in the sub-GeV range, with $480 \mathrm{MeV}$ positrons, was performed at the beam test facility of the INFNLaboratori Nazionali di Frascati [22]. Particles followed the bending of the crystal lattice, being guided by channeling phenomenon, and were deflected through the angles of about $10 \mathrm{mrad}$ over a length of $1 \mathrm{~mm}$ of silicon. Details of the Frascati experiment procedure, results, and comparison to simulations can be found in Ref. [22].

Our present study is encouraged by this experimental advance. Our aims are to find the crystal technique capabilities if applied to the sub-GeV range, and to understand the requirements to be fulfilled in order to make it more efficient. In the present paper we study only the physical aspects of the job, i.e., how much of the beam can be steered at a specific crystal angle, and what kind of bent crystal should be prepared.

In simulations, particle tracking through a curved crystal lattice is performed with the simulation code CATCH previously described in Ref. [23]. The code had earlier been successfully tested in crystal extraction experiments at CERN SPS [1], Tevatron [4], RHIC [9], and IHEP U-70 [6].

\section{CRYSTAL COLLIMATION}

The general idea of a crystal-assisted collimation [24] is that a crystal, serving as a primary element, gives the incident halo particles a bend of a few mrad providing a large impact parameter at some secondary element. The 
bent particles are absorbed there with a higher probability with a reduction of the backscattering problem. A radical solution might even be an extraction of bent particles to some external dump. Application of crystal collimation to $\mathrm{TeV}$ range accelerators is currently studied with great interest. In the energy range on the order of $1 \mathrm{GeV}$, the spallation neutron sources are one example where an efficient halo collimation is required for the protons accumulated in a ring [25].

A straightforward option would be to bend particles in a single pass through the bent crystal, at an angle of the order of $10 \mathrm{mrad}$. Protons with kinetic energy of $1 \mathrm{GeV}$ can move through silicon in the channeled states over $1-1.5 \mathrm{~mm}$. The bending radius $R$ must be greater than a critical one $\left(R_{c}=\right.$ $0.25 \mathrm{~cm}$ here); we take $R=10 \mathrm{~cm}$ as an example for our simulation. The angular distribution downstream of $1 \mathrm{~mm}$ long bent crystal is shown in Fig. 1. In this simulation, first we assumed that proton divergence at the incidence on the crystal was much narrower than the acceptance of the Si(110) crystal planes $2 \theta_{c}=0.25 \mathrm{mrad}$. Then, about $77 \%$ of the particles have exit angles greater than $1 \mathrm{mrad}$, and $30 \%$ of the total are bent at the full bending angle, $10 \mathrm{mrad}$. A further example in Fig. 1 repeats the simulation for the incident beam with divergence of $0.2 \mathrm{mrad}$. In that case $70 \%$ of the particles are bent more than $1 \mathrm{mrad}$, and $22 \%$ of the total are bent at $10 \mathrm{mrad}$.

Such a strong channeling effect would be a great help to collimation of the beams. Actual bending angles and the particle distribution downstream of a bent crystal can be designed as required to match some particular design of a cleaning system. The examples shown are for illustrative purposes only.

Notice that such a crystal deflector-producing a $10 \mathrm{mrad}$ bending over $1 \mathrm{~mm}$ of silicon-is already realized

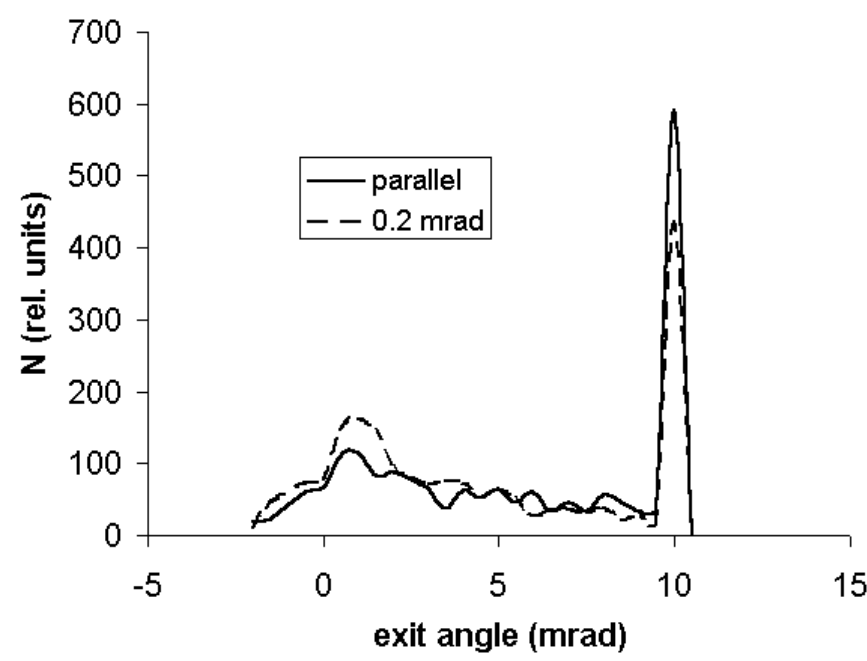

FIG. 1. Angular distribution of $1 \mathrm{GeV}$ protons downstream of the silicon crystal bent $10 \mathrm{mrad}$. For the parallel incident beam (solid line), and for the beam incident with divergence of $0.2 \mathrm{mrad}$ (dashed line). as described in Ref. [22] and tested recently at $480 \mathrm{MeV}$. The example of Fig. 1 shows that the available techniques are already suitable for efficient steering of sub-GeV beams with possible application for collimation.

The multiturn extraction, where halo particles can encounter the crystal several times while circulating in the ring [26], would be a rather interesting option due to a substantial increase of the extraction efficiency. This option is feasible if the crystal is short enough along the beam. This would reduce particle losses and scattering when they encounter the crystal, thus retaining the scattered particles in circulation.

In this option, we tried several bending angles from a fraction of mrad up to $5 \mathrm{mrad}$. Upon first encounter with a crystal, particles were allowed to circulate in the ring and have further encounters with the crystal or aperture (in that case the particle was removed). The lattice of the SNS 1$\mathrm{GeV}$ proton accumulation ring [25] (the linear transfer matrix was used with horizontal and vertical betatron tunes of $\nu_{x, y}=5.82 / 5.80$ and for the crystal position the horizontal betatron function of $10 \mathrm{~m}$ was selected) was selected as an example for simulation. The aperture limitation was imposed on the particle's angle at the crystal location: if its absolute value was greater than 0.5 (but less than 0.9 ) of the crystal bending angle, that particle was removed (considered lost at a collimator edge), so we counted only channeled particles that were steered at the proper angle.

The efficiency, for particles bent the full angle, was roughly independent of the chosen bending angle in the range studied, up to values of $5 \mathrm{mrad}$, with values of $85 \%-$ $90 \%$ with respect to the beam incident on the crystal. The unchanneled $10 \%-15 \%$ of the particles reach the collimator edge (the aperture); these unchanneled particles could be handled by traditional "amorphous" collimation.

The probability dependence on the crystal size with the bending angle of $3 \mathrm{mrad}$, for the unchanneled particles in the first encounter with crystal to be channeled on later encounters, is shown in Fig. 2. This probability is very high for the $50-\mu \mathrm{m}$ long crystals. Even the particles multiply scattered at first incidence in the crystal can be efficiently channeled on later turns and steered away. Because of multiple encounters, the initial divergence of particles at the crystal becomes not so critical. It can have a value close to the scattering angle along the crystal length. Crystal efficiency in multipass mode is defined mainly by the interplay of channeling and scattering processes in multiple encounters with a short crystal. The overall energy loss in multiple encounters with a crystal is within the nominal energy spread of the SNS beam, $\Delta E / E<4 \times$ $10^{-3}$ (rms).

For a typical particle, it takes 5 to 10 encounters with a crystal on average before the particle is channeled and extracted. A period from the moment of the first encounter with a crystal to the moment of the particle extraction from circulation in the ring corresponds to 100 turns. 


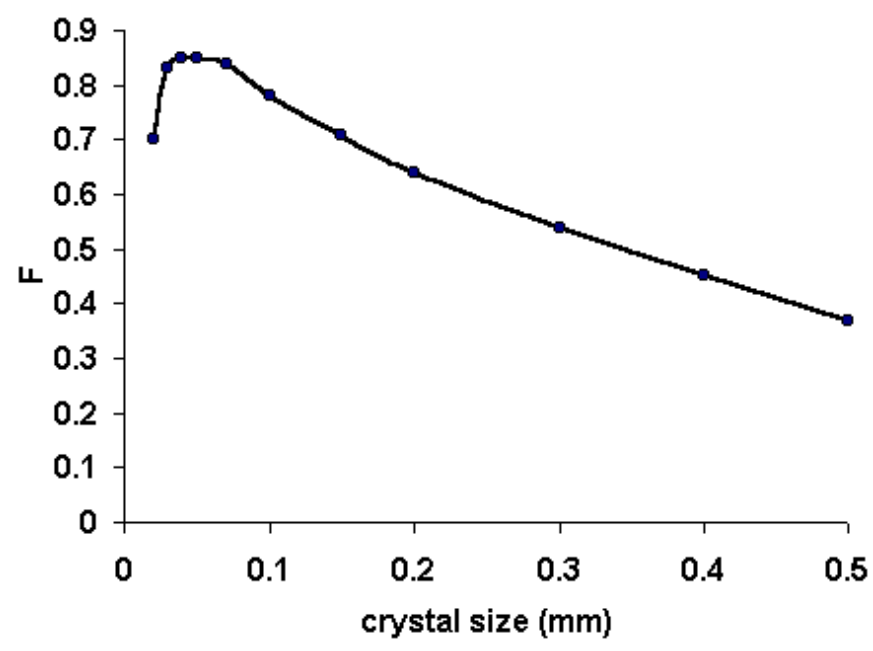

FIG. 2. (Color) Efficiency of crystal multipass scraping for the $1 \mathrm{GeV}$ particles that were not channeled on the first encounter, as a function of the crystal size along the beam.

Further simulation involving a realistic description of the machine and beam parameters would be necessary for realistic evaluation of a crystal-assisted scraping.

\section{SLOW EXTRACTION FROM MULTIHUNDRED MEV MACHINES}

The above-considered multipass channeling may be well suited for efficient slow extraction from medium-energy synchrotrons. The analytical theory of crystal extraction [27] predicts a reduction of the crystal size by a large factor, as the beam energy is lowered from values in multi-GeV down to $0.1-1 \mathrm{GeV}$. Tiny crystal size may permit a huge multiplicity of particle encounters with the crystal, and hence a very high overall efficiency of crystal channeling.

However, our simulations show that the extraction efficiency is limited by the multiple scattering to the aperture, although the loss due to nuclear interaction in the thin crystal at lower energy becomes insignificant (the aperture was set in these simulations at a value of 0.8 times the bending angle) as shown in Fig. 3. This factor is dominating with decreasing energy, Fig. 3. Nonetheless, the efficiency of crystal-assisted extraction is still $75 \%-80 \%$ at a few hundred MeV.

In these simulations we assumed the bending angle of $2 \mathrm{mrad}$ at any energy, and described particle revolutions in the ring by linear matrices. The accelerator lattice parameters were chosen from Ref. [28] for the synchrotron of the MedAustron hadron therapy center. We used these parameters throughout our studies just for illustrative purposes, despite the very broad energy range considered. The crystal size was scaled linearly with energy as suggested by the analytical theory [27]. Besides the two kinds of beam loss (i.e. nuclear interactions in the crystal and multiple scattering to the aperture), there was a dechanneling loss equal

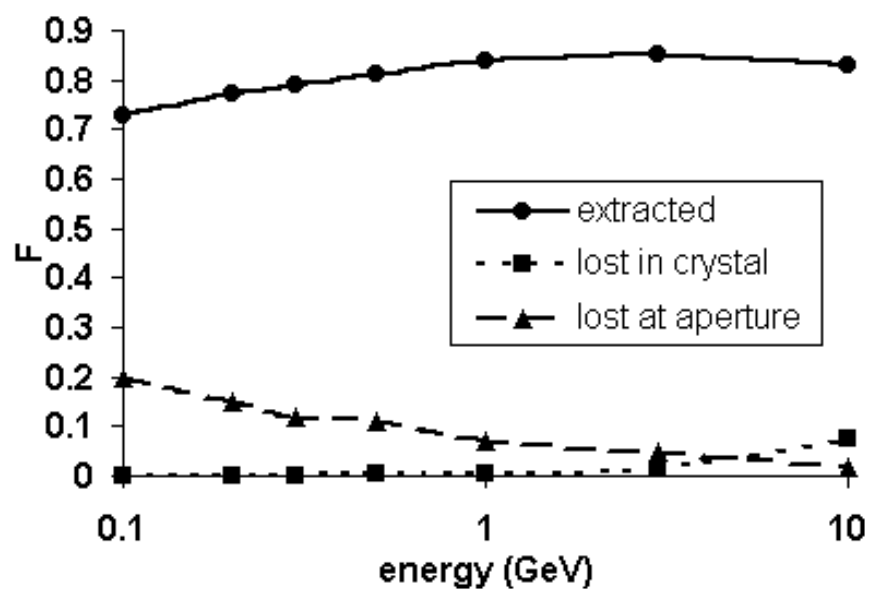

FIG. 3. Efficiency (dots) of crystal-assisted slow extraction, and beam lost in nuclear interactions in crystal (squares) and on the aperture (triangles), as functions of beam kinetic energy.

to $7 \%-8 \%$ of the beam in the cases considered, as shown in Fig. 3. This is due to the particles channeled only in a part of the crystal and, respectively, bent just part of the $2 \mathrm{mrad}$. The dechanneled particles are then lost somewhere on the machine apertures, similarly to multiply scattered particles.

The divergence of the extracted beam in the horizontal plane (plane of bending) is as small as $\pm 0.15 \mathrm{mrad} \times$ $(p v)^{-1 / 2}$ (full width, where $p v$ is beam momentum times velocity in $\mathrm{GeV}$ ) and defined by crystal properties only. In a vertical plane, the scattering in the encounters with crystal contributes (about $1 \mathrm{mrad}$ at $250 \mathrm{MeV}$ ) needs to be added quadratically to the initial divergence.

The experience in crystal extraction shows that this method may have additional benefits such as a flat time structure of the extracted beam [10].

Apart from crystal lattices, nanostructured material ${ }^{1}$ could be used in the future for channeling of accelerator beams [30], in particular, for producing microbeams and nanobeams [31] of very low emittance with applications in biology and medicine.

\section{CONCLUSIONS}

Efficient systems for slow extraction and halo scraping of beams at $0.1-10 \mathrm{GeV}$ accelerators and storage rings on the base of bent crystals could be designed. This is possible due to better understanding of beam channeling and scattering physics in bent crystal lattices, and progress in manufacturing of bent crystals. Beams of low emittances for applications in biology and medicine at medical synchrotrons could be produced as beam particles can be steered by the crystal beam channeling at the angles of several mrad with efficiencies of $70 \%-90 \%$.

\footnotetext{
${ }^{1}$ Let us recall that, in Frascati, our activity has been mainly focused on the study of nanostructures [29].
} 


\section{ACKNOWLEDGMENTS}

This work is supported in part by TARI Contract No. RII3-CT-2004-506078.

[1] H. Akbari et al., Phys. Lett. B 313, 491 (1993).

[2] G. Arduini et al., Phys. Rev. Lett. 79, 4182 (1997).

[3] V.M. Biryukov, Yu. A. Chesnokov, and V.I. Kotov, Crystal Channeling and its Application at High Energy Accelerators (Springer, Berlin, 1997).

[4] R. A. Carrigan et al., Phys. Rev. ST Accel. Beams 1, 022801 (1998).

[5] A. Baurichter et al., Nucl. Instrum. Methods Phys. Res., Sect. B 164, 27 (2000).

[6] A. G. Afonin et al., JETP Lett. 74, 55 (2001); 67, 781 (1998); Phys. Lett. B 435, 240 (1998).

[7] A. G. Afonin et al., Phys. Rev. Lett. 87, 094802 (2001).

[8] R. A. Carrigan et al., Phys. Rev. ST Accel. Beams 5, 043501 (2002).

[9] R. P. Fliller et al., Phys. Rev. ST Accel. Beams 9, 013501 (2006); Nucl. Instrum. Methods Phys. Res., Sect. B 234, 47 (2005); AIP Conf. Proc. 693, 192 (2004).

[10] A. G. Afonin et al., Nucl. Instrum. Methods Phys. Res., Sect. B 234, 14 (2005).

[11] V. Biryukov, Phys. Rev. E 52, 6818 (1995).

[12] E. Uggerhoj and U. I. Uggerhoj, Nucl. Instrum. Methods Phys. Res., Sect. B 234, 31 (2005); V. M. Biryukov, Phys. Rev. Lett. 74, 2471 (1995).

[13] V. M. Biryukov et al., Nucl. Instrum. Methods Phys. Res., Sect. B 234, 23 (2005); V. M. Biryukov and S. Bellucci, Nucl. Instrum. Methods Phys. Res., Sect. B 252, 7 (2006); H. H. Andersen, S. Bellucci, and V.M. Biryukov, Nucl. Instrum. Methods Phys. Res., Sect. B 252, 1 (2006); S. Bellucci and V. M. Biryukov, CERN Courier 46, 37 (2006).

[14] V.M. Biryukov, PAC Proceedings, New York, 1999, p. 1240 (hep-ex/0111034)].

[15] A. S. Vodopianov et al., JETP Lett. 30, 442 (1979).

[16] J. Back et al., Phys. Lett. 93B, 505 (1980).

[17] V. A. Andreev et al., JETP Lett. 36, 415 (1982).

[18] V.V. Avdeichikov et al., Joint Institute for Nuclear Research (Dubna) 1, 3(R) (1984).

[19] M. B. H. Breese, Nucl. Instrum. Methods Phys. Res., Sect. B 132, 540 (1997); M.B.H. Breese, d.D. G. Kerckhove, P. J.M. Smulders, W. M. Arnold Bik, and D. O. Boerma, Nucl. Instrum. Methods Phys. Res., Sect. B 171, 387 (2000).

[20] S. Bellucci et al., Phys. Rev. Lett. 90, 034801 (2003); S. Bellucci et al., Phys. Rev. ST Accel. Beams 7, 023501 (2004); V. T. Baranov et al., JETP Lett. 82, 562 (2005).

[21] V.T. Baranov et al., Nucl. Instrum. Methods Phys. Res., Sect. B 252, 32 (2006); S. Bellucci and V. M. Biryukov, CERN Courier 44, 19 (2004); A. G. Afonin et al., Nucl.
Instrum. Methods Phys. Res., Sect. B 234, 122 (2005).

[22] S. Bellucci et al., JETP Lett. 83, 95 (2006); S. Bellucci, S. Balasubramanian, A. Grilli, F. Micciulla, A. Raco, A. Popov, V. Baranov, V. Biryukov, Y. Chesnokov, and V. Maisheev, Nucl. Instrum. Methods Phys. Res., Sect. B 252, 3 (2006).

[23] V. Biryukov, Phys. Rev. E 51, 3522 (1995); CERN SL/ Note 93-74 AP, 1993; Nucl. Instrum. Methods Phys. Res., Sect. B 117, 463 (1996).

[24] D. Trbojevic et al., EPAC Proceedings, Stockholm, 1998, p. 2146 (hep-ex/0111021); V.M. Biryukov, physics/ 0307027.

[25] J. Wei, Rev. Mod. Phys. 75, 1383 (2003).

[26] V. M. Biryukov, Nucl. Instrum. Methods Phys. Res., Sect. B 53, 202 (1991); A. M. Taratin et al., Nucl. Instrum. Methods Phys. Res., Sect. B 58, 103 (1991).

[27] V. M. Biryukov, Nucl. Instrum. Methods Phys. Res., Sect. B 153, 461 (1999); EPAC Proceedings, Stockholm, 1998, p. 2091.

[28] M. Benedikt, Nucl. Instrum. Methods Phys. Res., Sect. A 539, 25 (2005).

[29] S. Bellucci et al., 19th International Conference on X-ray and Inner-Shell Processes, edited by A. Bianconi (American Institute of Physics, Melville, New York, 2003), p. 355; M. Bottini, L. Tautz, H. Huynh, E. Monosov, N. Bottini, M.I. Dawson, S. Bellucci, and T. Mustelin, Chem. Commun. 6, 758 (2005); M. Bottini, S. Bruckner, K. Nika, N. Bottini, S. Bellucci, A. Magrini, A. Bergamaschi A, and T. Mustelin, Toxicol. Lett. 160, 121 (2006); M. Bottini, A. Magrini, A. Di Venere, S. Bellucci, M.I. Dawson, N. Rosato, A. Bergamaschi, and T. Mustelin, J. Nanosci. Nanotechnol. 6, 1381 (2006); M. Bottini, C. Balasubramanian, M. I. Dawson, A. Bergamaschi, S. Bellucci, T. Mustelin, J. Phys. Chem. B 110, 831 (2006); Z. Y. Wu et al., Appl. Phys. Lett. 80, 2973 (2002); C. Balasubramanian et al., Chem. Phys. Lett. 383, 188 (2004); S. Bellucci and S. B. Dabagov, J. Phys. Condens. Matter 15, 3171 (2003); S. Bellucci, Phys. Status Solidi C 2, 34 (2005); J. Phys. Condens. Matter 18, S1967 (2006); Yu. F. Zhukovskii, A. I. Popov, C. Balasubramanian, and S. Bellucci, J. Phys. Condens. Matter 18, S2045 (2006); L. Galfetti, L. T. De Luca, F. Severini, L. Meda, G. Marra, M. Marchetti, M. Regi, and S. Bellucci, J. Phys. Condens. Matter 18, S1991 (2006); C. Balasubramanian, S. Bellucci, G. Cinque, A. Marcelli, M. Cestelli Guidi, M. Piccinini, A. Popov, A. Soldatov, and P. Onorato, J. Phys. Condens. Matter 18, S2095 (2006).

[30] S. Bellucci et al., Nucl. Instrum. Methods Phys. Res., Sect. B 202, 236 (2003); V. M. Biryukov and S. Bellucci, Phys. Lett. B 542, 111 (2002); Nucl. Instrum. Methods Phys. Res., Sect. B 230, 619 (2005).

[31] S. Bellucci et al., Phys. Rev. ST Accel. Beams 6, 033502 (2003); V. Biryukov, S. Bellucci, and V. Guidi, Nucl. Instrum. Methods Phys. Res., Sect. B 231, 70 (2005). 